J. Korean Math. Soc. 50 (2013), No. 1, pp. 111-125

http://dx.doi.org/10.4134/JKMS.2013.50.1.111

\title{
ON THE PROBABILITY OF RUIN IN A CONTINUOUS RISK MODEL WITH DELAYED CLAIMS
}

\author{
Wei Zou And Jie-HuA Xie
}

\begin{abstract}
In this paper, we consider a continuous time risk model involving two types of dependent claims, namely main claims and by-claims. The by-claim is induced by the main claim and the occurrence of byclaim may be delayed depending on associated main claim amount. Using Rouché's theorem, we first derive the closed-form solution for the Laplace transform of the survival probability in the dependent risk model from an integro-differential equations system. Then, using the Laplace transform, we derive a defective renewal equation satisfied by the survival probability. For the exponential claim sizes, we present the explicit formula for the survival probability. We also illustrate the influence of the model parameters in the dependent risk model on the survival probability by numerical examples.
\end{abstract}

\section{Introduction}

The classical Cramer-Lundberg model describing the surplus process of an insurance portfolio relies on the assumption of independence among claim sizes and between claim sizes and claim inter-occurrence times. However, in practice this assumption is often too restrictive and there is a need for more general models where the independence assumptions can be relaxed. Recently, various results have been obtained concerning the asymptotic behaviour of the probability of ruin for dependent claims. In the case of light-tailed claim sizes, Albrecher and Boxma [1] considered the case in which the distribution of a claim interval is controlled by the previous claim size through a mechanism that if the claim size exceeds a random level the next claim interval will follow one type of distribution, if not, it will follow another type of distribution. Their model depicts a common sense that when a certain kind of catastrophe is big enough, people will pay more attention to it and so the time until the next occurrence is longer, and vice versa. Albrecher and Teugels [2] and Boudreault et al. [4] considered a particular dependence structure among the inter-claim

Received January 10, 2012

2010 Mathematics Subject Classification. 60H10, 62P05.

Key words and phrases. continuous time risk model, survival probability, delayed claim, Laplace transform. 
time and subsequent claim size. Furthermore, Zhang and Yang [15, 16] have studied the risk models with dependence between inter-claim times and claim sizes.

In fact, insurance claims may be delayed due to various reasons. This phenomenon may happen in reality. For a catastrophe such as an earthquake or a rain-storm, it is very likely that there exist other insurance claims after the immediate ones. Since the work by Waters and Paratriandafylou [8], risk models with this special feature have been discussed by many authors in the literature. For example, Boogaert and Haezendonck [3] studied the mathematical properties of a liability process with settling delay within the framework of an economics environment. Yuen and Guo [13] studied a compound binomial model with delayed claims and obtained recursive formulas for the finite time survival probabilities. Xiao and Guo [9] also studied this risk model. They derived an upper bound for the ruin probability. Xie and Zou [10] studied an extension to the risk model proposed in Yuen and Guo [13]. Yuen, Guo and $\mathrm{Ng}$ [14] studied a risk model with delayed claims, in which the time of delay for the occurrence of a by-claim is assumed to be exponentially distributed. Macci [7] presented a sample path large deviation principle for the delayed claims risk model presented in Yuen, Guo and Ng [14]. Xie and Zou [11] first considered the risk model with delayed claims and a constant dividend barrier in a financial market driven by a time-homogeneous Markov chain. The expected discounted dividend payments prior to ruin were derived. Xie and Zou [12] considered the compound Poisson risk model with delayed claims and proved that the ruin probability for this risk model decreases as the probability of the delay of by-claims is increasing.

Note that, all risk models described in the paragraph above relied on the assumption that the probability of delay of each claim is constant and independent of claim amounts. However, there exist many real-world situations for which such an assumption is inappropriate. For instance, for a line of business covering damages due to motor accidents, there are different kinds of claim in a serious motor accident, such as car damage and injury. It is very likely that the bigger the claim amount for car damage become, the greater odds of the claim for injury would be delayed. Hence, it is an interesting problem to consider the risk model with delayed claims, in which the probability of delay of claims depends on previous claim size.

Motivated by this idea and by the dependent risk model developed in Albrecher and Boxma [1], in this paper, we consider a continuous time risk model with two types of individual claims and the two types of claim have different distributions of severity. In this risk model, there will be a claim, namely main claim, at every jump time of the number process. Each main claim induces another type of claim called by-claim. Moreover, the occurrence of the by-claim may be delayed depending on associated main claim amount. If the main claim amount is less than a threshold variable, the main claim and its associated byclaim occur simultaneously; if the main claim amount is larger than or equal 
to a threshold variable, the occurrence of the by-claim will be delayed. We also assume that the occurrence of the delayed by-claim is independent of the occurrence of next main claim.

The risk model proposed in this paper is a generalization of compound Poisson risk model with delayed claims. We show that, the explicit expression for the survival probability in this risk model can be obtained. The work of this paper can also be seen as a complement to the work of Xie and Zou [12] and extend their results by taking the relevancy between the delay of by-claims and the main claim amounts into account.

It is obvious that the incorporation of the randomness of delay of the claim makes the problem more interesting. It also complicates the analysis of the model. Our aim is to give an exact representation for the survival probability in the delayed claims risk model. The paper is structured as follows: we describe the risk model in detail and define the surplus process of this model in Section 2. In Section 3, we derive an integro-differential equations system for survival probabilities. Both of the survival probabilities with zero initial surplus and the Laplace transforms of the survival probabilities are obtained in Section 4. Then the defective renewal equation for the survival probability is obtained and exact representation for the solution of this equation is derived through an associated compound geometric distribution in Section 5. The explicit formulas for survival probabilities are obtained when the claim amounts from both classes are exponentially distributed in Section 6. In Section 6, we also illustrate the influence of the model parameters in the dependent risk model on the survival probability by numerical examples.

\section{The model}

Here, we consider a continuous time model which involves two types of insurance claims, namely the main claims and the by-claims. Let the aggregate main claims process be a compound Poisson process and let $\{N(t) ; t \geq 0\}$ be the corresponding Poisson claim number process, with intensity $\lambda$. Its jump times are denoted by $\left\{T_{i}\right\}_{i \geq 1}$ with $T_{0}=0$. The main claim amounts $\left\{Y_{i}\right\}_{i \geq 1}$ are assumed to be independent and identically distributed (i.i.d.) positive random variables with common distribution $F$. Let $\left\{X_{i}\right\}_{i>1}$ be the by-claim amounts, assumed to be i.i.d. positive random variables with common distribution $G$. The main claim amounts and by-claim amounts are independent and their means are denoted by $\mu_{F}$ and $\mu_{G}$.

In this risk model, we assume the claim occurrence process to be of the following type: there will be a main claim $Y_{i}$ at every epoch $T_{i}$ of the Poisson process and the main claim $Y_{i}$ will induce a by-claim $X_{i}$. Moreover, if the main claim amount $Y_{i}$ is less than a threshold $B_{i}$, the by-claim $X_{i}$ and its associated main claim $Y_{i}$ occur simultaneously; if the main claim amount $Y_{i}$ is larger than or equal to the threshold $B_{i}$, the occurrence of the by-claim $X_{i}$ is delayed to $T_{i+1}$. If the occurrence of the by-claim $X_{i}$ is delayed to $T_{i+1}$, then the delayed 
by-claim $X_{i}$ and main claim $Y_{i+1}$ occur simultaneously. The quantities $\left\{B_{i}\right\}_{i \geq 1}$ are assumed to be i.i.d. random variables with common distribution $B$.

In this setup, the surplus process $U(t)$ of this risk model is defined as

$$
U(t)=u+c t-\sum_{i=1}^{N(t)} Y_{i}-R(t)
$$

where $u$ is the initial capital, $c$ is the constant rate per unit time at which the premiums are received, and $R(t)$ is the sum of all by-claims $X_{i}$ that occurred before time $t$.

One of the key quantities in the risk model is the survival probability, or non-ruin probability, denoted by $\Phi(u)$ as a function of $u \geq 0$, which is the probability that the surplus of the insurer is always above zero, namely,

$$
\Phi(u)=\operatorname{Pr}(U(t) \geq 0 ; \quad \text { for all } t \geq 0) .
$$

The corresponding ruin probability is then $\Psi(u)=1-\Phi(u)$, which is the probability that the surplus of the insurer is below zero at some time. The survival probability (ruin probability) can be used to provide an early warning system for the guidance of an insurance project.

The expectation of the aggregate claims at time $t$ is given by

$$
\mathrm{E}\left[\sum_{i=1}^{N(t)} Y_{i}+R(t)\right]=\lambda t \mu_{F}+\lambda t \mu_{G}-\operatorname{Pr}\left(Y_{1} \geq B_{1}\right) \mu_{G}\left(1-e^{-\lambda t}\right) .
$$

Thus in order to ensure that premium rate exceeds the net claim rate and guarantee that ruin does not occur almost surely, we assume the following positive safely loading condition holds, i.e.,

$$
\lambda\left(\mu_{F}+\mu_{G}\right)<c .
$$

With other things being the same, we consider a slight change in the risk model. Instead of having one main claim $Y_{1}$ and a by-claim $X_{1}$ with probability $\operatorname{Pr}\left(Y_{1}<B_{1}\right)$ at the first epoch $T_{1}$, another by-claim $X$ is added at the first epoch $T_{1}$, i.e., by-claim $X$ and main claim $Y_{1}$ occur at $T_{1}$ simultaneously. Hence, the corresponding surplus process $U_{1}(t)$ of this auxiliary risk model is defined as

$$
U_{1}(t)=u+c t-\sum_{i=1}^{N(t)} Y_{i}-R(t)-X,
$$

where $X$ denotes the another by-claim amount, $U_{1}(0)=u$. Assume that $X$ and $\left\{X_{i}\right\}_{i \geq 1}$ are i.i.d. positive random variables.

This surplus process is similar to (2.1) except for the subtraction of the byclaim random variable $X$. Denote the corresponding survival probability for this auxiliary model by $\Phi_{1}(u)$ which is very useful in the derivation of $\Phi(u)$. From the definitions of (2.1) and (2.3), it is easy to see that $\Phi(u) \geq \Phi_{1}(u)$. 


\section{System of integro-differential equations}

We are interested in the survival probability $\Phi(u)$. Consider what will happen at the first epoch $T_{1}$. Obviously there will be a main claim $Y_{1}$. The main claim will induce a by-claim $X_{1}$. If $Y_{1}<B_{1}$, the by-claim $X_{1}$ also occurs at the first epoch $T_{1}$, the surplus process $U(t)$ will renew itself with different initial reserve. The probability of this event is $\operatorname{Pr}\left(Y_{1}<B_{1}\right)$. If $Y_{1} \geq B_{1}$, the occurrence of the by-claim $X_{1}$ will be delayed to $T_{2}$, i.e., the delayed by-claim $X_{1}$ and main claim $Y_{2}$ occur simultaneously. In this case, $U(t)$ will not renew itself but transfer to the auxiliary model described in the paragraph above. The probability of this event is $\operatorname{Pr}\left(Y_{1} \geq B_{1}\right)$. Remember that the survival probability of the auxiliary model is $\Phi_{1}(u)$. Taking what happened at the first epoch $T_{1}$ into account, we can set up the following equation for $\Phi(u)$ :

$$
\begin{aligned}
\Phi(u)= & \int_{0}^{\infty} \lambda e^{-\lambda t} \iint_{0<x+y<u+c t} \operatorname{Pr}\left(y<B_{1}\right) \Phi(u+c t-x-y) \mathrm{d} F(y) \mathrm{d} G(x) \mathrm{d} t \\
& +\int_{0}^{\infty} \lambda e^{-\lambda t} \int_{0}^{u+c t} \operatorname{Pr}\left(y \geq B_{1}\right) \Phi_{1}(u+c t-y) \mathrm{d} F(y) \mathrm{d} t .
\end{aligned}
$$

With the auxiliary model, similar analysis gives

$$
\begin{aligned}
\Phi_{1}(u)= & \int_{0}^{\infty} \lambda e^{-\lambda t} \iint_{0<x+y<u+c t} \operatorname{Pr}\left(y<B_{1}\right) \Phi(u+c t-x-y) \mathrm{d} F(y) \mathrm{d} G * G(x) \mathrm{d} t \\
& +\int_{0}^{\infty} \lambda e^{-\lambda t} \iint_{0<x+y<u+c t} \operatorname{Pr}\left(y \geq B_{1}\right) \Phi_{1}(u+c t-y) \mathrm{d} F(y) \mathrm{d} G(x) \mathrm{d} t
\end{aligned}
$$

where $*$ denotes the operation of convolution.

Setting $s=u+c t$ in (3.1), (3.2) and differentiating with respect to $u$, we get the following system of integro-differential equations:

$$
\begin{aligned}
& c \frac{\mathrm{d} \Phi(u)}{\mathrm{d} u}-\lambda \Phi(u) \\
& +\lambda \iint_{0<x+y<u} \operatorname{Pr}\left(y<B_{1}\right) \Phi(u-x-y) \mathrm{d} F(y) \mathrm{d} G(x) \\
& +\lambda \int_{0}^{u} \operatorname{Pr}\left(y \geq B_{1}\right) \Phi_{1}(u-y) \mathrm{d} F(y)=0
\end{aligned}
$$

and

$$
\begin{aligned}
& c \frac{\mathrm{d} \Phi_{1}(u)}{\mathrm{d} u}-\lambda \Phi_{1}(u) \\
& +\lambda \iint_{0<x+y<u} \operatorname{Pr}\left(y<B_{1}\right) \Phi(u-x-y) \mathrm{d} F(y) \mathrm{d} G * G(x) \\
& +\lambda \iint_{0<x+y<u} \operatorname{Pr}\left(y \geq B_{1}\right) \Phi_{1}(u-x-y) \mathrm{d} F(y) \mathrm{d} G(x)=0 .
\end{aligned}
$$




\section{Laplace transforms}

Henceforth, we focus on our interest on the survival probabilities $\Phi(u)$ and $\Phi_{1}(u)$. Their Laplace transforms can be derived as follows.

For $\operatorname{Re} s \geq 0$, we define

$$
\begin{aligned}
\tilde{\chi}_{1}(s) & =\int_{y=0}^{\infty} \exp (-s y) \chi_{1}(y) \mathrm{d} y=\mathrm{E}\left[\exp (-s F) 1_{(F \geq B)}\right] \\
& =\int_{y=0}^{\infty} \exp (-s y) B(y) \mathrm{d} F(y) \\
\tilde{\chi}_{2}(s) & =\int_{y=0}^{\infty} \exp (-s y) \chi_{2}(y) \mathrm{d} y=\mathrm{E}\left[\exp (-s F) 1_{(F<B)}\right] \\
& =\int_{y=0}^{\infty} \exp (-s y)(1-B(y)) \mathrm{d} F(y) \\
\tilde{b}_{(s)} & =\int_{0}^{\infty} \exp (-s y) \mathrm{d} F(y) \\
\tilde{b}_{1}(s) & =\int_{0}^{\infty} \exp (-s x) \mathrm{d} G(x) \\
\tilde{b}_{2}(s) & =\int_{0}^{\infty} \exp (-s x) \mathrm{d} G * G(x) .
\end{aligned}
$$

Note that $\tilde{b}(s)=\tilde{\chi}_{1}(s)+\tilde{\chi}_{2}(s)$ and $\tilde{b}_{2}(s)=\left(\tilde{b}_{1}(s)\right)^{2}$.

We also define the Laplace transforms of $\Phi(u)$ and $\Phi_{1}(u)$ as

$$
\tilde{\Phi}(s)=\int_{0}^{\infty} \exp (-s u) \Phi(u) \mathrm{d} u ; \quad \tilde{\Phi}_{1}(s)=\int_{0}^{\infty} \exp (-s u) \Phi_{1}(u) \mathrm{d} u .
$$

Taking Laplace transforms of (3.3) and (3.4) and making some simplifications, we obtain

$$
\begin{gathered}
c(-\Phi(0)+s \tilde{\Phi}(s))-\lambda \tilde{\Phi}(s)+\lambda \tilde{\Phi}(s) \tilde{\chi}_{2}(s) \tilde{b}_{1}(s)+\lambda \tilde{\Phi}_{1}(s) \tilde{\chi}_{1}(s)=0, \\
c\left(-\Phi_{1}(0)+s \tilde{\Phi}_{1}(s)\right)-\lambda \tilde{\Phi}_{1}(s)+\lambda \tilde{\Phi}(s) \tilde{\chi}_{2}(s) \tilde{b}_{2}(s)+\lambda \tilde{\Phi}_{1}(s) \tilde{\chi}_{1}(s) \tilde{b}_{1}(s)=0,
\end{gathered}
$$

which can further be simplified to

$$
\begin{gathered}
\tilde{\Phi}(s)=\frac{c \Phi(0) \alpha(s)-\lambda c \Phi_{1}(0) \tilde{\chi}_{1}(s)}{(c s-\lambda)\left\{c s-\lambda+\lambda \tilde{b}(s) \tilde{b}_{1}(s)\right\}}, \\
\tilde{\Phi}_{1}(s)=\frac{c \Phi_{1}(0) \beta(s)-\lambda c \Phi(0) \tilde{b}_{2}(s) \tilde{\chi}_{2}(s)}{(c s-\lambda)\left\{c s-\lambda+\lambda \tilde{b}(s) \tilde{b}_{1}(s)\right\}}
\end{gathered}
$$

where

$$
\alpha(s)=c s-\lambda+\lambda \tilde{b}_{1}(s) \tilde{\chi}_{1}(s) ; \quad \beta(s)=c s-\lambda+\lambda \tilde{b}_{1}(s) \tilde{\chi}_{2}(s) .
$$

In order to obtain $\tilde{\Phi}(s)$ and $\tilde{\Phi}_{1}(s)$, for the further sake of deriving $\Phi(u)$ and $\Phi_{1}(u)$, we only need to find $\Phi(0)$ and $\Phi_{1}(0)$. 
Note that the denominators on the right-hand side of (4.1) and (4.2) coincide. Denoting the denominator as

$$
\triangle(s)=(c s-\lambda)\left\{c s-\lambda+\lambda \tilde{b}(s) \tilde{b}_{1}(s)\right\}
$$

we have

$$
\triangle(s)=c s\left\{(c s-2 \lambda)+\lambda \tilde{b}(s) \tilde{b}_{1}(s)+\frac{\lambda^{2}-\lambda^{2} \tilde{b}(s) \tilde{b}_{1}(s)}{c s}\right\} .
$$

Since $\lim _{u \rightarrow+\infty} \Phi(u)=1$, by final-value theorem of Laplace transform, we have $\lim _{s \rightarrow 0} s \tilde{\Phi}(s)=1$, then

$$
\begin{aligned}
1 & =\lim _{s \rightarrow 0} s \frac{c \Phi(0) \alpha(s)-\lambda c \Phi_{1}(0) \tilde{\chi}_{1}(s)}{(c s-\lambda)\left\{c s-\lambda+\lambda \tilde{b}(s) \tilde{b}_{1}(s)\right\}} \\
& =\lim _{s \rightarrow 0} s \frac{c \Phi(0)\left(c s-\lambda+\lambda \tilde{b}_{1}(s) \tilde{\chi}_{1}(s)\right)-\lambda c \Phi_{1}(0) \tilde{\chi}_{1}(s)}{\triangle(s)} \\
& =\frac{\Phi(0)\left(\tilde{b}_{1}(0) \tilde{\chi}_{1}(0)-1\right)-\Phi_{1}(0) \tilde{\chi}_{1}(0)}{-2+\tilde{b}(0) \tilde{b}_{1}(0)-\frac{\left.\lambda\left(\tilde{b}(s) \tilde{b}_{1}(s)\right)^{\prime}\right|_{s=0}}{c}} \\
& =\frac{\Phi(0) \tilde{\chi}_{2}(0)+\Phi_{1}(0) \tilde{\chi}_{1}(0)}{1-\frac{\lambda}{c}\left(\mu_{F}+\mu_{G}\right)}
\end{aligned}
$$

where $\tilde{b}^{\prime}(0)=-\mu_{F}, \tilde{b}_{1}^{\prime}(0)=-\mu_{G}$, and $\tilde{b}_{2}^{\prime}(0)=-2 \mu_{G}$. (4.3) is an equation for $\Phi(0)$ and $\Phi_{1}(0)$. In order to derive the explicit results for $\tilde{\Phi}(s)$ and $\tilde{\Phi}_{1}(s)$, we must obtain a second equation for $\Phi(0)$ and $\Phi_{1}(0)$. Using Rouché's theorem, we have:

Proposition 4.1. Equation $\Delta(s)=0$ has exactly one positive real root, say $\sigma=\lambda / c$, on the right half complex plane.

Proof. Define $l(s)=c s-\lambda+\lambda \tilde{b}(s) \tilde{b}_{1}(s)$, then $\Delta(s)=(c s-\lambda) l(s)$. It is easy to check that $l(0)=0$ and $\lim _{s \rightarrow+\infty} l(s)=+\infty$. Also, for $s \geq 0$,

$$
l^{\prime}(s)=c+\lambda \tilde{b}^{\prime}(s) \tilde{b}_{1}(s)+\lambda \tilde{b}(s) \tilde{b}_{1}^{\prime}(s)>c-\lambda\left(\mu_{F}+\mu_{G}\right)>0,
$$

then $l(s)$ is an increasing function of s. Hence, $l(s)=0$ has no positive real root. Noting that $\lambda / c$ is one positive real root of equation $\Delta(s)=0$, we conclude that equation $\Delta(s)=0$ has exactly one positive real root, say, $\sigma=\lambda / c$.

Now, we prove that $\sigma=\lambda / c$ is the exactly one positive real root of equation $\Delta(s)=0$ on the right half complex plane. In order to prove this result, we only need to prove that $l(s)=0$ has no positive real root on the right half complex plane. For $\delta>0$, assume that $l_{\delta}(s)=c s-\lambda-\delta+\lambda \tilde{b}(s) \tilde{b}_{1}(s)$. If $s$ is on the half circle: $|z|=r(r>0)$ and $\operatorname{Re}(z) \geq 0$ on the complex plane, $|c s-\lambda-\delta|>\lambda=\lambda \tilde{b}(0) \tilde{b}_{1}(0)>\left|\lambda \tilde{b}(s) \tilde{b}_{1}(s)\right|$ for $r$ is sufficiently large, while if $s$ is on the imaginary axis, $\operatorname{Re}(s)=0,|c s-\lambda-\delta|>\lambda \geq\left|\lambda \tilde{b}(s) \tilde{b}_{1}(s)\right|$. This implies on the boundary of the contour enclosed by the half circle and the imaginary 
axis, that $|c s-\lambda-\delta|>\left|\lambda \tilde{b}(s) \tilde{b}_{1}(s)\right|$. We conclude, by Rouché's theorem, that on the right half complex plane, the number of roots of the equation $l_{\delta}(s)=0$ equals the number of roots of the equation $c s-\lambda-\delta=0$. Furthermore, the latter has exactly one root on the right half complex plane. It follows that $l_{\delta}(s)=0$ has exactly one positive real root, say, $\rho(\delta)$, on the right half complex plane.

Finally, it is easy to see that $\rho(\delta) \rightarrow 0$ as $\delta \rightarrow 0^{+}$from the expression of $l_{\delta}(s)$. According to $\lim _{\delta \rightarrow 0^{+}} l_{\delta}(s)=l(s)$, we can conclude that $l(s)=0$ has no root with positive real part on the right half plane.

It follows from all above that equation $\Delta(s)=0$ has exactly one positive real root $\sigma=\lambda / c$ on the right half complex plane. This completes the proof.

Since $\tilde{\Phi}(s)$ and $\tilde{\Phi}_{1}(s)$ are analytic functions for $\boldsymbol{R e}(s) \geq 0, \sigma$ must also be a zero of the numerators of (4.1) and (4.2). In both cases this yields the same relation between $\Phi(0)$ and $\Phi_{1}(0)$, namely

$$
\Phi(0)=\frac{\Phi_{1}(0)}{\tilde{b}_{1}(\sigma)} .
$$

Combining (4.3) and (4.4), we can get the constants:

$$
\begin{gathered}
\Phi(0)=\frac{\lambda \tilde{\chi}_{1}(\sigma)\left(c-\lambda \mu_{F}-\lambda \mu_{G}\right)}{-\lambda c \tilde{\chi}_{1}(0)+c^{2} \sigma \tilde{\chi}_{1}(0)+c \lambda \tilde{\chi}_{1}(\sigma) \tilde{b}_{1}(\sigma) \tilde{\chi}_{1}(0)+\lambda c \tilde{\chi}_{1}(\sigma) \tilde{\chi}_{2}(0)}, \\
\Phi_{1}(0)=\frac{\left(c-\lambda \mu_{F}-\lambda \mu_{G}\right)\left(c \sigma-\lambda+\lambda \tilde{\chi}_{1}(\sigma) \tilde{b}_{1}(\sigma)\right)}{-\lambda c \tilde{\chi}_{1}(0)+c^{2} \sigma \tilde{\chi}_{1}(0)+c \lambda \tilde{\chi}_{1}(\sigma) \tilde{b}_{1}(\sigma) \tilde{\chi}_{1}(0)+\lambda c \tilde{\chi}_{1}(\sigma) \tilde{\chi}_{2}(0)} .
\end{gathered}
$$

Remark. 1. Let $B=+\infty$, i.e., $\operatorname{Pr}(Y<B)=1$. Then $\tilde{\chi}_{1}(s)=0$ and $\tilde{\chi}_{2}(s)=\tilde{b}(s)$. In this case, each main claim and its associated by-claim occur simultaneously. Actually, the risk model given by (2.1) is the classic compound Poisson risk model and the claim amounts are $\left\{Y_{i}+X_{i}\right\}_{i \geq 1}$. Then Eq.(4.1) can be simplified as

$$
\tilde{\Phi}(s)=\frac{c \Phi(0)}{c s-\lambda+\lambda \tilde{b}(s) \tilde{b}_{1}(s)},
$$

which is the Laplace transform of the non-ruin probability in the classic compound Poisson risk model. Similarly, since $\lim _{u \rightarrow+\infty} \Phi(u)=1$, by final value theorem of Laplace transform, we have $\lim _{s \rightarrow 0} s \tilde{\Phi}(s)=1$, i.e.,

$$
1=\lim _{s \rightarrow 0} s \frac{c \Phi(0)}{s\left(c+\frac{\lambda\left(\tilde{b}(s) \tilde{b}_{1}(s)-1\right)}{s}\right)}=\frac{c \Phi(0)}{c-\lambda\left(\mu_{F}+\mu_{G}\right)},
$$

then we obtain

$$
\Phi(0)=\frac{c-\lambda\left(\mu_{F}+\mu_{G}\right)}{c},
$$

which is the well-known formula for the non-ruin probability with zero initial capital in the classic compound Poisson risk model. 
2. Let $B$ be a constant, i.e., $\operatorname{Pr}(Y \geq B)=\theta$. Then $\tilde{\chi}_{1}(s)=\theta \tilde{b}(s)$ and $\tilde{\chi}_{2}(s)=(1-\theta) \tilde{b}(s)$. In this case, each main claim and its associated by-claim occur simultaneously with probability $1-\theta$, or the occurrence of the by-claim may be delayed with probability $\theta$. Actually, the risk model given by (2.1) is the compound Poisson risk model with delayed claims studied by Xie and Zou [12]. Then Eq.(4.1) can be simplified as

$$
\tilde{\Phi}(s)=\frac{c \Phi(0)\left(c s-\lambda+\lambda \theta \tilde{b}(s)\left(\tilde{b}_{1}(s)-\tilde{b}_{1}\left(\frac{\lambda}{c}\right)\right)\right)}{(c s-\lambda)\left(c s-\lambda+\lambda \tilde{b}(s) \tilde{b}_{1}(s)\right)} .
$$

This equation is consistent with Eq.(4.1) in Xie and Zou [12]. Similarly, since $\lim _{u \rightarrow+\infty} \Phi(u)=1$, by final value theorem of Laplace transform, we have $\lim _{s \rightarrow 0} s \tilde{\Phi}(s)=1$, i.e.,

$$
\begin{aligned}
1 & =\lim _{s \rightarrow 0} s \frac{c \Phi(0)\left(c s-\lambda+\lambda \theta \tilde{b}(s)\left(\tilde{b}_{1}(s)-\tilde{b}_{1}\left(\frac{\lambda}{c}\right)\right)\right)}{(c s-\lambda)\left(c s-\lambda+\lambda \tilde{b}(s) \tilde{b}_{1}(s)\right)} \\
& =\lim _{s \rightarrow 0} s \frac{c \Phi(0)\left(c s-\lambda+\lambda \theta \tilde{b}(s)\left(\tilde{b}_{1}(s)-\tilde{b}_{1}\left(\frac{\lambda}{c}\right)\right)\right)}{c s\left\{(c s-2 \lambda)+\lambda \tilde{b}(s) \tilde{b}_{1}(s)+\frac{\lambda^{2}-\lambda^{2} \tilde{b}(s) \tilde{b}_{1}(s)}{c s}\right\}} \\
& =\frac{\Phi(0)\left(-1+\theta \tilde{b}(0)\left(\tilde{b}_{1}(0)-\tilde{b}_{1}\left(\frac{\lambda}{c}\right)\right)\right)}{\left\{-2+\tilde{b}(0) \tilde{b}_{1}(0)-\frac{\left.\lambda\left(\tilde{b}(s) \tilde{b}_{1}(s)\right)^{\prime}\right|_{s=0}}{c}\right\}} \\
& =\frac{\Phi(0)\left(-1+\theta\left(1-\tilde{b}_{1}\left(\frac{\lambda}{c}\right)\right)\right)}{-1+\frac{\lambda}{c}\left(\mu_{F}+\mu_{G}\right)}
\end{aligned}
$$

then we obtain

$$
\Phi(0)=\frac{1-\frac{\lambda}{c}\left(\mu_{F}+\mu_{G}\right)}{1-\theta\left(1-\tilde{b}_{1}\left(\frac{\lambda}{c}\right)\right)},
$$

which is the formula for the survival probability with zero initial capital in the compound Poisson risk model with delayed claims.

\section{Defective renewal equations for survival probabilities}

In this section, our goal is to show that the survival probabilities also satisfy a defective renewal equation in the continuous time risk model with delayed claims. To identify the form of this defective renewal equation, we first analyse the Laplace transform of $\Phi(u)$.

As in Dickson and Hipp [5], we define an operator $\Gamma_{r}$ of a real-valued function $f$, with respect to a complex number $r$, to be

$$
\Gamma_{r} f(x)=\int_{x}^{\infty} e^{-r(y-x)} f(y) \mathrm{d} y, \quad x \geq 0 .
$$


It is clear that the Laplace transform of $f, \tilde{f}(s)$, can be expressed as $\Gamma_{s} f(0)$, and that for distinct $r_{1}$ and $r_{2}$,

$$
\Gamma_{r_{1}} \Gamma_{r_{2}} f(x)=\Gamma_{r_{2}} \Gamma_{r_{1}} f(x)=\frac{\Gamma_{r_{1}} f(x)-\Gamma_{r_{2}} f(x)}{r_{2}-r_{1}}, \quad x \geq 0 .
$$

If $r_{1}=r_{2}=r$

$$
\Gamma_{r_{1}} \Gamma_{r_{2}} f(x)=\int_{x}^{\infty}(y-x) e^{-r(y-x)} f(y) \mathrm{d} y, \quad x \geq 0 .
$$

The properties for this operator can be found in Dickson and Hipp [5].

Lemma 5.1. The Laplace transform $\tilde{\Phi}(s)$ of the survival probability satisfies

$$
\tilde{\Phi}(s)=\frac{\lambda \Gamma_{s} \Gamma_{0} b * b_{1}(0)}{c} \tilde{\Phi}(s)+\Phi(0)\left(\frac{1}{s}-\frac{\lambda \tilde{\chi}_{1}(s)}{c s} \Gamma_{s} \Gamma_{\sigma} b_{1}(0)\right) .
$$

Proof. Substituting (4.4) into (4.1), we can get

$$
\begin{aligned}
\tilde{\Phi}(s) & =\frac{c \Phi(0)\left(c s-\lambda+\lambda \tilde{\chi}_{1}(s)\left(\tilde{b}_{1}(s)-\tilde{b}_{1}(\sigma)\right)\right)}{(c s-\lambda)\left\{c s-\lambda+\lambda \tilde{b}(s) \tilde{b}_{1}(s)\right\}} \\
& =\frac{c s(s-\sigma) \Phi(0)\left(\frac{c}{s}+\frac{\lambda \tilde{\chi}_{1}(s)}{s} \frac{\tilde{b}_{1}(s)-\tilde{b}_{1}(\sigma)}{s-\sigma}\right)}{c s(s-\sigma)\left(c-\frac{\lambda-\lambda \tilde{b}(s) \tilde{b}_{1}(s)}{s}\right)} \\
& =\frac{\Phi(0)\left(\frac{c}{s}-\frac{\lambda \tilde{\chi}_{1}(s)}{s} \Gamma_{s} \Gamma_{\sigma} b_{1}(0)\right)}{c-\lambda \Gamma_{s} \Gamma_{0} b * b_{1}(0)},
\end{aligned}
$$

which leads to (5.1). This completes the proof.

Using Lemma 5.1, we are now in a position to derive the defective renewal equation for $\Phi(u)$.

Theorem 5.1. $\Phi(u)$ satisfies the following defective renewal equation

$$
\Phi(u)=\frac{\lambda\left(\mu_{F}+\mu_{G}\right)}{c} \int_{0}^{u} \Phi(u-y) \vartheta(y) \mathrm{d} y+z(u),
$$

where

$$
\vartheta(y)=\frac{\Gamma_{0} b * b_{1}(y)}{\mu_{F}+\mu_{G}}, \quad z(u)=\Phi(0)\left(1-\frac{\lambda}{c} * \chi_{1} * \Gamma_{\sigma} b_{1}(u)\right) .
$$

Proof. Inverting the Laplace transform in (5.1), one finds

$$
\begin{aligned}
\Phi(u) & \left.=\frac{\lambda}{c} \int_{0}^{u} \Phi(u-y) \Gamma_{0} b * b_{1}(y) \mathrm{d} y+\Phi(0)\left(1-\frac{\lambda}{c} * \chi_{1} * \Gamma_{\sigma} b_{1}(u)\right)\right) \\
& =\frac{\lambda \Gamma_{0} \Gamma_{0} b * b_{1}(0)}{c} \int_{0}^{u} \Phi(u-y) \frac{\Gamma_{0} b * b_{1}(y)}{\Gamma_{0} \Gamma_{0} b * b_{1}(0)} \mathrm{d} y+z(u) \\
& =\frac{\lambda\left(\mu_{F}+\mu_{G}\right)}{c} \int_{0}^{u} \Phi(u-y) \frac{\Gamma_{0} b * b_{1}(y)}{\mu_{F}+\mu_{G}} \mathrm{~d} y+z(u),
\end{aligned}
$$


which corresponds to (5.2).

For (5.2) to be a defective renewal equation, it remains to show that

$$
\frac{\lambda\left(\mu_{F}+\mu_{G}\right)}{c}<1
$$

The inequality is the positive safely loading condition (2.2). Thus, we complete the proof.

Now, we define an associated compound geometric distribution function $K(u)=1-\bar{K}(u)$ by

$$
\bar{K}(u)=\frac{\zeta}{1+\zeta} \sum_{n=1}^{\infty}\left(\frac{1}{1+\zeta}\right)^{n} \bar{Z}^{* n}(u), \quad u \geq 0,
$$

where $\zeta=\left(c-\lambda\left(\mu_{F}+\mu_{G}\right)\right) /\left[\lambda\left(\mu_{F}+\mu_{G}\right)\right], \bar{Z}^{* n}(u)$ is the tail of the $n$-fold convolution of $Z(u)=1-\bar{Z}(u)=\int_{0}^{u} \vartheta(y) \mathrm{d} y$. Explicit solutions of the defective renewal Eq.(5.2) can be derived directly by applying Theorem 2.1 of Lin and Willmot [6].

Proposition 5.1. The survival probability $\Phi(u)$ satisfying the defective renewal equation (5.2) can be expressed as

$$
\Phi(u)=\frac{1}{\zeta} \int_{0}^{u}[1-\bar{K}(u-y)] \mathrm{d} H(y)+\frac{H(0)}{\zeta}[1-\bar{K}(u)],
$$

or

$$
\Phi(u)=\frac{1}{\zeta} \int_{0}^{u} H(u-y) \mathrm{d} K(y)+\frac{1}{1+\zeta} H(u),
$$

where $H(u)=c z(u) /\left[\lambda\left(\mu_{F}+\mu_{G}\right)\right]$.

Proof. The proof is straightforward using Theorem 2.1 of Lin and Willmot [6] and Eq.(5.2).

\section{Explicit results for exponential claim size distributions}

We now consider the case where both the claim sizes are exponentially distributed, with distribution functions $F \sim \operatorname{Exp}(\nu)$ and $G \sim \operatorname{Exp}(\omega)$, respectively, where $\nu=\frac{1}{\mu_{F}}$ and $\omega=\frac{1}{\mu_{G}}$. Then we have

$$
\tilde{b}(s)=\frac{\nu}{\nu+s}, \quad \tilde{b}_{1}(s)=\frac{\omega}{\omega+s}, \quad \tilde{b}_{2}(s)=\frac{\omega^{2}}{(\omega+s)^{2}} .
$$

For the special case $B \sim \operatorname{Exp}(\mu)$ we obtain

$$
\tilde{\chi}_{2}(s)=\int_{y=0}^{\infty} \exp (-s y) \exp (-\mu y) \mathrm{d} F(y)=b(s+\mu), \quad \tilde{\chi}_{1}(s)=b(s)-b(s+\mu) .
$$

So we can derive

$$
\tilde{\chi}_{2}(s)=\frac{\nu}{\nu+s+\mu} ; \quad \tilde{\chi}_{1}(s)=\frac{\nu}{\nu+s}-\frac{\nu}{\nu+s+\mu} ;
$$


and $\sigma$ in (4.4) is the unique solution $s$ with $\boldsymbol{\operatorname { R e }}(s)>0$ of

$$
(c s-\lambda)\left(c s-\lambda+\lambda\left(\frac{\omega}{\omega+s}\right)\left(\frac{\nu}{\nu+s}\right)\right)=0 .
$$

By solving this equation, we obtain four roots:

$$
\begin{aligned}
\sigma_{1} & =0 \\
\sigma_{2} & =\frac{\lambda}{c}, \\
\sigma_{3} & =\frac{\lambda-c \nu-c \omega-\sqrt{(-\lambda+c \nu+c \omega)^{2}-4 c(-\lambda \nu-\lambda \omega+c \nu \omega)}}{2 c}, \\
\sigma_{4} & =\frac{\lambda-c \nu-c \omega+\sqrt{(-\lambda+c \nu+c \omega)^{2}-4 c(-\lambda \nu-\lambda \omega+c \nu \omega)}}{2 c} .
\end{aligned}
$$

The positive relative security loading condition (2.2) implies that only $\sigma_{2}$ has positive real part. Hence, $\sigma=\lambda / c$ is the only zero with positive real part on the right half plane. It proves the correctness of Proposition 4.1.

Taking the expressions of $\tilde{b}_{1}(s), \tilde{b}_{2}(s), \tilde{\chi}_{1}(s)$ and $\tilde{\chi}_{2}(s)$ into (4.1) and (4.2), we have

$\tilde{\Phi}(s)=\frac{-\Phi_{1}(0) \lambda c \mu \nu(s+\omega)+\Phi(0) c^{2} s(s+\nu)(s+\mu+\nu)(s+\omega)-\Phi(0) \lambda c f_{1}(s)}{s(c s-\lambda)(s+\mu+\nu)(c(s+\nu)(s+\omega)-\lambda(s+\nu+\omega))}$,

and

$$
\tilde{\Phi}_{1}(s)=\frac{c(s+\nu)\left(-\Phi(0) \lambda \nu \omega^{2}+\Phi_{1}(0)(s+\omega) f_{2}(s)\right)}{s(c s-\lambda)(s+\mu+\nu)(c(s+\nu)(s+\omega)-\lambda(s+\nu+\omega))}
$$

where

$$
\begin{aligned}
& f_{1}(s)=s^{3}+s^{2}(\mu+2 \nu+\omega)+s(\mu(\nu+\omega)+\nu(\nu+2 \omega))+\nu^{2} \omega, \\
& f_{2}(s)=c s(s+\mu+\nu)(s+\omega)-\lambda\left(s^{2}+\mu \omega+s(\mu+\nu+\omega)\right) .
\end{aligned}
$$

Substituting $\Phi(0)$ and $\Phi_{1}(0)$ into $\tilde{\Phi}(s)$ and $\tilde{\Phi}_{1}(s)$ and taking the inverse Laplace transforms, we can derive explicit expressions for $\Phi(u)$ and $\Phi_{1}(u)$,

$$
\begin{aligned}
\Phi(u)= & +\frac{e^{-u(\mu+\nu)} \lambda \mu(c \nu \omega-\lambda(\nu+\omega))}{(\lambda \nu+c(\mu+\nu) \omega)(\lambda(\mu-\omega)+c \mu(\mu+\nu-\omega))} \\
& +\frac{e^{-\frac{u(-\lambda+c(\nu+\omega)+\rho)}{2 c}}\{\lambda(\mu+\nu)(\lambda(\nu+\omega)-c \nu \omega)(\vartheta-(\lambda+c(\mu+\nu))(\lambda+c \omega) \rho)\}}{c \nu \omega(\lambda \nu+c(\mu+\nu) \omega) \rho\left\{\lambda^{2}+c \lambda(\mu+\nu)-c^{2}((\nu-\omega) \omega+\mu(\nu+\omega))-(\lambda+c(\mu-\omega)) \rho\right\}} \\
& -\frac{e^{-\frac{u(-\lambda+c(\nu+\omega)-\rho)}{2 c}}\{\lambda(\mu+\nu)(\lambda(\nu+\omega)-c \nu \omega)(\vartheta+(\lambda+c(\mu+\nu))(\lambda+c \omega) \rho)\}}{c \nu \omega(\lambda \nu+c(\mu+\nu) \omega) \rho\left\{\lambda^{2}+c \lambda(\mu+\nu)-c^{2}((\nu-\omega) \omega+\mu(\nu+\omega))+(\lambda+c(\mu-\omega)) \rho\right\}},
\end{aligned}
$$




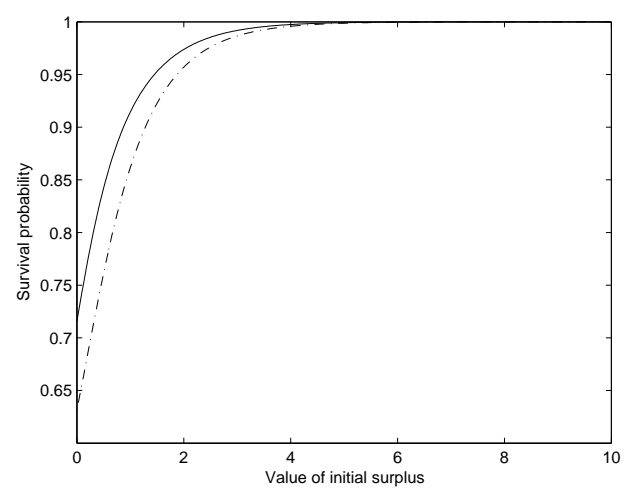

FiguRE 1. Survival probabilities $\Phi(u)$ (solid line) and $\Phi_{1}(u)$ (dashed line) in Example 1.

and

(6.2)

$$
\begin{aligned}
\Phi_{1}(u)= & -\frac{e^{-u(\mu+\nu)} \lambda \mu \omega(c \nu \omega-\lambda(\nu+\omega))}{(\mu+\nu-\omega)(\lambda \nu+c(\mu+\nu) \omega)(\lambda(\mu-\omega)+c \mu(\mu+\nu-\omega))} \\
& -\frac{e^{-\frac{u(-\lambda+c(\nu) \omega)+\rho)}{2 c}}\{\lambda(\mu+\nu)(\lambda(\nu+\omega)-c \nu \omega)(\vartheta-(\lambda+c(\mu+\nu))(\lambda+c \omega) \rho)\}}{\nu(\lambda \nu+c(\mu+\nu) \omega) \rho\left\{\left(\lambda^{2}+c \mu(\lambda-c \nu)\right) \rho-\left(\lambda^{3}+c^{3} \mu \nu(\nu-\omega)+c \lambda(c \omega(\mu-2 \nu)+\lambda(\mu+\nu+\omega))\right)\right\}} \\
& -\frac{e^{-\frac{u(-\lambda+c(\nu+\omega)-\rho)}{2 c}}\{\lambda(\mu+\nu)(\lambda(\nu+\omega)-c \nu \omega)(\vartheta+(\lambda+c(\mu+\nu))(\lambda+c \omega) \rho)\}}{\nu(\lambda \nu+c(\mu+\nu) \omega) \rho\left\{\left(\lambda^{2}+c \mu(\lambda-c \nu)\right) \rho+\left(\lambda^{3}+c^{3} \mu \nu(\nu-\omega)+c \lambda(c \omega(\mu-2 \nu)+\lambda(\mu+\nu+\omega))\right)\right\}} \\
& +\frac{e^{-u \omega}(\mu+\nu)(\nu-\omega)(c \nu \omega-\lambda(\nu+\omega))}{\nu(\mu+\nu-\omega)(\lambda \nu+c(\mu+\nu) \omega)}, \quad u \geq 0,
\end{aligned}
$$

where

$$
\begin{aligned}
\rho= & \sqrt{\lambda^{2}+c^{2}(\nu-\omega)^{2}+2 c \lambda(\nu+\omega)} \\
\vartheta= & \lambda^{3}-c^{3}(\mu-\nu)(\nu-\omega) \omega+c^{2} \lambda\left(\nu(\mu+\nu)+(2 \mu+\nu) \omega+\omega^{2}\right) \\
& +c \lambda^{2}(\mu+2(\nu+\omega))
\end{aligned}
$$

Example 1. Let $\lambda=1, c=2.5, B \sim \operatorname{Exp}(2.8), F \sim \operatorname{Exp}(2), G \sim \operatorname{Exp}(3)$. The positive relative security loading condition (2.2) is obviously fulfilled. Moreover, we have $\sigma=0.4$. Accordingly, explicit expressions for $\Phi(u)$ and $\Phi_{1}(u)$, when the claim sizes from both classes are exponentially distributed, are given by (6.1) and (6.2),

$$
\begin{aligned}
\Phi(u)= & 1+0.0594228 e^{-4.8 u}-0.0754902 e^{-3.43578 u}-0.268143 e^{-1.16422 u}, \\
\Phi_{1}(u)= & 1-0.0990379 e^{-4.8 u}+0.519688 e^{-3.43578 u}-0.438194 e^{-1.16422 u} \\
& -0.350877 e^{-3 u}
\end{aligned}
$$


Figure 1 shows the survival probabilities $\Phi(u)$ and $\Phi_{1}(u)$ in Example 1, for different values of $u \in[0,10]$. One can see from the graph that both $\Phi(u)$ and $\Phi_{1}(u)$ are strictly increasing functions of $u$. They are increasing sharply when $u$ is small (between 0 and approximately 2) and turn to be flatter when $u$ increases. Moreover $\Phi(u)$ is always greater than $\Phi_{1}(u)$.

Example 2. In this example, we show the influence of the threshold $B$ on the survival probabilities. Let $\lambda=1, c=2, F \sim \operatorname{Exp}(1.5), G \sim \operatorname{Exp}(1)$, $B \sim \operatorname{Exp}(\mu)$. The positive relative security loading condition (2.2) is obviously fulfilled. Table 1 illustrates the influence of the coefficient $\mu$ of threshold $B$ on the survival probabilities. We see from Table 1 that the coefficient $\mu$ of threshold $B$ has a significant effect on the non-ruin probabilities. Table 1 also shows the fact that non-ruin probability is an increasing function of $\mu$.

TABLE 1. Survival probabilities $\Phi(u)$ in Example 2.

\begin{tabular}{lllllll}
\hline$u \backslash \mu$ & 0.5 & 1 & 1.5 & 2 & 2.5 & 3 \\
\hline$u=0$ & 0.181818 & 0.192308 & 0.199999 & 0.205882 & 0.210526 & 0.214286 \\
$u=1$ & 0.279932 & 0.293465 & 0.302962 & 0.309952 & 0.315291 & 0.319492 \\
$u=2$ & 0.370831 & 0.383514 & 0.392104 & 0.398296 & 0.402970 & 0.406625 \\
$u=3$ & 0.449991 & 0.461160 & 0.468662 & 0.474058 & 0.478129 & 0.481315 \\
$u=4$ & 0.519022 & 0.528788 & 0.535341 & 0.540055 & 0.543613 & 0.546396 \\
$u=5$ & 0.579344 & 0.587883 & 0.593613 & 0.597734 & 0.600845 & 0.603279 \\
$u=6$ & 0.632092 & 0.639559 & 0.644569 & 0.648174 & 0.650895 & 0.653024 \\
$u=7$ & 0.678223 & 0.684754 & 0.689137 & 0.692289 & 0.694669 & 0.696531 \\
$u=8$ & 0.718570 & 0.724282 & 0.728115 & 0.730872 & 0.732954 & 0.734582 \\
$u=9$ & 0.753858 & 0.758854 & 0.762206 & 0.766438 & 0.766438 & 0.767862 \\
$u=10$ & 0.784721 & 0.789091 & 0.792023 & 0.794132 & 0.795724 & 0.796969 \\
\hline
\end{tabular}

\section{Concluding remarks}

In this paper, we study a continuous risk model with delayed claims. In this risk model, there will be a main claim $Y_{i}$ at every epoch $T_{i}$ of the Poisson process and the main claim $Y_{i}$ will induce a by-claim $X_{i}$. Moreover, if the main claim amount $Y_{i}$ is less than a threshold $B_{i}$, the by-claim $X_{i}$ and its associated main claim $Y_{i}$ occur simultaneously; if the main claim amount $Y_{i}$ is larger than or equal to the threshold $B_{i}$, the occurrence of the by-claim $X_{i}$ is delayed to $T_{i+1}$. If the occurrence of the by-claim $X_{i}$ is delayed to $T_{i+1}$, then the delayed by-claim $X_{i}$ and main claim $Y_{i+1}$ occur simultaneously. The quantities $\left\{B_{i}\right\}_{i \geq 1}$ are assumed to be i.i.d. non-negative random variables. We show how to apply the Laplace transform to this dependent risk model. The results obtained in this paper show (although the risk process is neither a compound renewal nor a compound Poisson one) that the survival probability satisfies the defective renewal equation. The explicit results for survival probabilities are derived when the claims are exponentially distributed. Some examples and numerical illustrations are also given. 
Acknowledgements. This research is supported by the Natural Science Foundation of China under Grant No. 11201217.

\section{References}

[1] H. Albrecher and O. J. Boxma, A ruin model with dependence between claim sizes and claim intervals, Insurance Math. Econom. 35 (2004), no. 2, 245-254.

[2] H. Albrecher and J. L. Teugels, Exponential behavior in the presence of dependence in risk theory, J. Appl. Probab. 43 (2006), no. 1, 257-273.

[3] P. Boogaert and J. Haezendonck, Delay in claim settlement, Insurance Math. Econom. 8 (1989), no. 4, 321-330.

[4] M. Boudreault, H. Cossette, D. Landrault, and E. Marceau, On a risk model with dependence between interclaim arrivals and claim sizes, Scand. Actuar. J. 2006 (2006), no. $5,265-285$.

[5] D. C. M. Dickson and C. Hipp, On the time to ruin for Erlang(2) risk processes, Insurance Math. Econom. 29 (2001), no. 3, 333-344.

[6] X. S. Lin and G. E. Willmot, Analysis of a defective renewal equation arising in ruin theory, Insurance Math. Econom. 25 (1999), no. 1, 63-84.

[7] C. Macci, Large deviations for risk models in which each main claim induces a delayed claim, Stochastics 78 (2006), no. 2, 77-89.

[8] H. R. Waters and A. Papatriandafylou, Ruin probabilities allowing for delay in claims settlement, IInsurance Math. Econom. 4 (1985), no. 2, 113-122.

[9] Y. T. Xiao and J. Y. Guo, The compound binomial risk model with time-correlated claims, Insurance Math. Econom. 41 (2007), no. 1, 124-133.

[10] J. H. Xie and W. Zou, Ruin probabilities of a risk model with time-correlated claims, Journal of the Graduate School of the Chinese Academy of Sciences 25 (2008), 319-326.

[11] _ Expected present value of total dividends in a delayed claims risk model under stochastic interest rates, Insurance Math. Econom. 46 (2010), no. 2, 415-422.

[12] - On the expected discounted penalty function for the compound Poisson risk model with delayed claims, J. Comput. Appl. Math. 235 (2011), no. 8, 2392-2404.

[13] K. C. Yuen and J. Y. Guo, Ruin probabilities for time-correlated claims in the compound binomial model, Insurance Math. Econom. 29 (2001), no. 1, 47-57.

[14] K. C. Yuen, J. Y. Guo, and K. W. Ng, On ultimate ruin in a delayed-claims risk model, J. Appl. Probab. 42 (2005), no. 1, 163-174.

[15] Z. M. Zhang and H. Yang, On a risk model with stochastic premiums income and dependence between income and loss, J. Comput. Appl. Math. 234 (2010), no. 1, 44-57.

[16] , Gerber-Shiu analysis in a perturbed risk model with dependence between claim sizes and interclaim times, J. Comput. Appl. Math. 235 (2011), no. 5, 1189-1204.

WeI Zou

Department of SCIENCE

NAnChang Institute of Technology

NANChang 330099, P. R. China

E-mail address: zouwei@nit.edu.cn

JiE-HuA XIE

Department of SCience

NanChang Institute of Technology

NanChang 330099, P. R. China

E-mail address: jhxie@nit.edu.cn 